\title{
Small Bowel Adenocarcinoma Simulating Superior Mesenteric Artery Syndrome (SMAS): A Case Report
}

Othmane El Yamine, Nassima Fakhiri, Dounia Benkiran, Rachid Boufettal, Amal Hajri, Driss Erguibi, Saad Rifki Jai, Farid Chehab, Nabila El Gasmi, Fatima Zahrae Cheikhna, Mohamed Tahiri, and Wafaa Badre

\section{ABSTRACT}

The adenocarcinoma of the small intestine is a rare tumor of all gastrointestinal cancers often occurs on a predisposing ground we report the case of a 40-year-old patient chronic smoker has 1 pack year weaned 2 years ago, alcoholic weaned 5 years ago whose symptomatology dates back to 3 months by the appearance of epigastralgia and vomiting post prandial, Complicated by a high occlusive syndrome made of post prandial vomiting with abdominal pain all evolving in a context of alteration of general state made the patient was addressed to the service of digestive cancer surgery and liver transplantation of CHU Ibn Rochd Casablanca.

The patient had an abdominal CT scan which showed duodenojejunal distension with incarceration of an undistended loop at the level of the aorto-mesenteric clamp.

The patient was sent to the operating room in emergency, he underwent a segmental Greco-Resection of $15 \mathrm{~cm}$ taking away a jejunal mass under laparoscopy with extra corporal anastomosis latero-lateral jejuno-jejunal with at the exploration one notes the presence of a tumoral mass of $3 \mathrm{~cm}$ mobile at the level of the 2 nd loop jejunal stenosing responsible for a jejunal distension upstream measuring $4 \mathrm{~cm}$ in diameter.

The anatomopathological study of the specimen showed a moderately differentiated and invasive adenocarcinoma, classified as pT3N1Mx.

The postoperative follow-up was marked by a deep venous thrombosis involving the ileo-femoral-popliteal axis of the ilio-femoral-popliteal trunk on day 2 postoperatively, for which the patient was put on low molecular weight Heparin at a curative dose with compression stockings and monitoring.

The patient had resumed transit in the form of gas on postoperative day 3 , with a correct assessment, and was declared discharged on postoperative day 6.

The patient's file was discussed in a multidisciplinary consultation meeting and the decision was to undergo adjuvant chemotherapy and oesogastroduodal fibroscopy (FOGD) and colonoscopy to look for predisposing diseases.

Keywords: adenocarcinoma, Artery Syndrome, occlusive syndrome, Superior Mesenteric, the small intestine.
Submitted : Jun 06, 2021

Published : July 20, 2021

ISSN: $2593-8339$

DOI: $10.24018 /$ ejmed.2021.3.4.922

\section{Othmane El Yamine}

Department of Visceral Surgery, CHU Ibn Rochd, Casablanca, Morocco.

(e-mail: othmane.elyamine@gmail.com) Nassima Fakhiri*

Department of Visceral Surgery, CHU Ibn Rochd, Casablanca, Morocco.

(e-mail: nassima.fakhiri@gmail.com)

Dounia Benkiran

Department of Visceral Surgery, CHU Ibn Rochd, Casablanca, Morocco.

(e-mail: d.viscerale@gmail.com) Rachid Boufettal

Department of Visceral Surgery, CHU Ibn Rochd, Casablanca, Morocco.

(e-mail: racbouf@hotmail.fr)

Amal Hajri

Department of Visceral Surgery CHU Ibn Rochd Casablanca, Morocco.

(e-mail: amalhajri.282@gmail.com)

Driss Erguibi

Department of Visceral Surgery CHU Ibn Rochd Casablanca, Morocco.

(e-mail: driss.erguibi@gmail.com)

Saad Rifki Jai

Department of Visceral Surgery CHU Ibn Rochd Casablanca, Morocco.

(e-mail: saadjai@yahoo.fr)

Farid Chehab

Department of Visceral Surgery, CHU Ibn Rochd, Casablanca, Morocco.

(e-mail: farid_chehab@yahoo.fr)

Nabila EI Gasmi

Department of Visceral Surgery, CHU Ibn Rochd, Casablanca, Morocco.

(e-mail: elgasminabila@gmail.com)

Fatima Zahrae Cheikhna

Department of Gastroenterology, CHU Ibn Rochd, Casablanca, Morocco.

(e-mail: zcheikhna02@gmail.com)

Mohamed Tahiri

Department of Gastroenterology, CHU Ibn

Rochd, Casablanca, Morocco.

(e-mail: docteurtahirimohamed@yahoo.fr)

Wafaa Badre

Department of Gastroenterology, CHU Ibn Rochd, Casablanca, Morocco.

(e-mail: wafaa6badre6dr@gmail.com)

*Corresponding Author 


\section{INTRODUCTION}

Small bowel Adenocarcinoma is defined by the invasion of neoplastic glandular structures through the muscularis mucosae. It is a rare tumor accounting for less than $2 \%$ of all gastrointestinal cancers [1]. The annual incidence of adenocarcinomas is increasing with male predisposition. It is often associated with a number of predisposing conditions such as Peutz-Jeghers polyposis, celiac disease or Crohn's disease [2].

The preferred location is the duodenum followed by the jejunal and ileal locations [3].

These cancers pose a problem of diagnosis because of the absence of specific signs, the diagnosis is often late explaining the high frequency of advanced stages [4].

It is often revealed by melena, abdominal mass and Koenig syndrome or occlusive syndrome [5]. We report a case of a 40-year-old patient presenting an occlusive syndrome due to a small bowel adenocarcinoma simulating an aortomesenteric compression syndrome.

\section{CASE REPORT}

This is a 40-years-old patient with a history of chronic smoking and alcohol consumption. Three months ago, he experienced epigastralgia and post-prandial vomiting, for which he had undergone an Oeso-gastroduodenal fibroscopy which showed a crenelated aspect of the duodenal mucosa and an erythematous gastritis, the symptomatology worsened after 2 weeks by the onset of an occlusive syndrome leading to postprandial vomiting with abdominal pain, and weight loss. The patient was referred to the department of digestive cancer surgery and liver transplantation of CHU Ibn Rochd Casablanca.

Physical examination revealed a soft abdomen with epigastric tenderness, no mass was palpated. BMI: $18 \mathrm{~kg} / \mathrm{m}^{2}$. Laboratory tests showed anaemia, hemoglobin at $8.4 \mathrm{~g} / \mathrm{dl}$, leukocytes at $5290 / \mathrm{mm}^{3} \mathrm{X}$, platelets at $252,000 / \mathrm{IU}$, Creatinine at $7.7 \mathrm{mg} / \mathrm{l}$, Urea at $0.15 \mathrm{~g} / \mathrm{l}$, Natremia at $135 \mathrm{mEq} / 1$, Kalaemia at $3.9 \mathrm{mEq} / 1$, Albumin at $29 \mathrm{~g} / 1$, and a correct haemostasis result. Abdominal X-ray (AXR) showed hailic hydroaeric levels.

The patient underwent an abdominal CT-scan which showed duodeno-jejunal distension with incarceration of an undistended loop at the level of the ortho-mesenteric clamp, aorto-mesenteric space evaluated at $7 \mathrm{~mm}$, with irregular circumferential wall thickening of a jejunal loop. the junction between left flank and epigastrium measuring $14 \mathrm{~mm}$ in maximum thickness with distension of the upstream loops reaching $61 \mathrm{~mm}$ in diameter (Fig. 1), (Fig. 2).

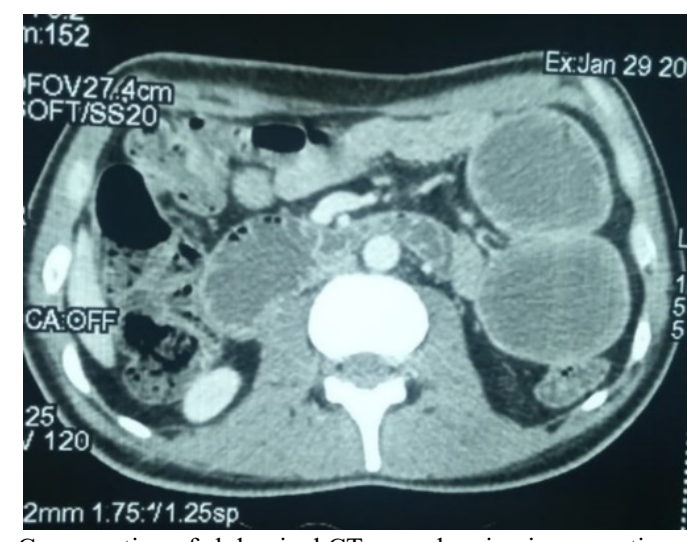

Fig. 1. Cross section of abdominal CT scan showing incarceration of an undistended loop at the level of the aorto-mesenteric pincer.

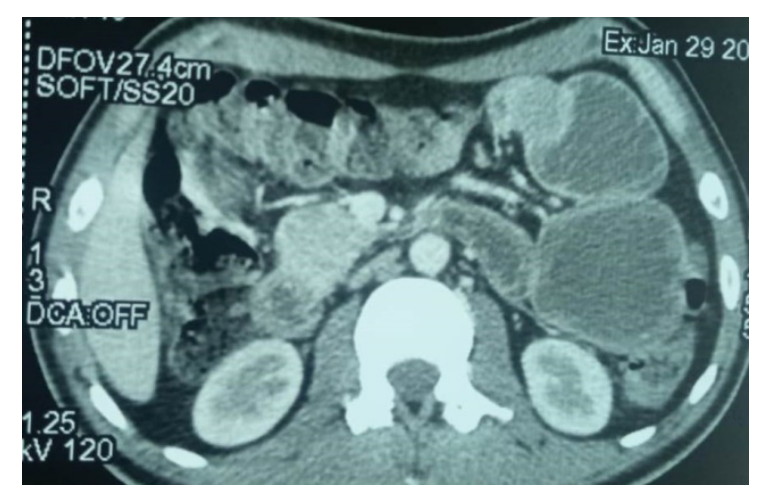

Fig. 2. Cross section of abdominal CT scan showing thickening irregular circumferential parietal wall of a jejunal loop.

An urgent laparoscopy revealed a $3 \mathrm{~cm}$ mobile stenosing jejunal tumor. Accordingly, a segmental hail of $15 \mathrm{~cm}$ with the jejunal mass was resected. There was no evidence of metastatic lesions in the peritoneum or liver during intraoperative inspection.

The histological study of the resected mass showed an invasive and moderately differenciated adenocarcinoma, measuring $3.5 \mathrm{~cm}$ with metastasis to three ouf of five lymph node. Free surgical margins were achieved. The tumor was staged as pT3N1Mx.

Two days later, the patient had a postoperative deep vein thrombosis of the ileo-femoro-popliteal axis and was put on low molecular weight heparin with compression stockings.

Thoracic CT scan was free of metastasis (Fig. 5). ACE was 1ng/ml and CA19-9 was $3 \mathrm{IU} / \mathrm{ml}$.

Oesogastroduodal fibroscopy (FOGD) and colonoscopy were normal.

A multidisciplinary team decided an adjuvant chemotherapy.

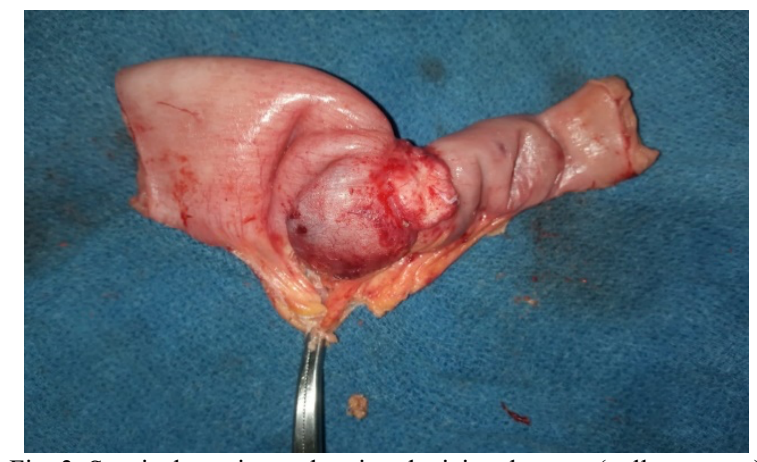

Fig. 3. Surgical specimen showing the jejunal tumor (yellow arrow) responsible for the distension. 


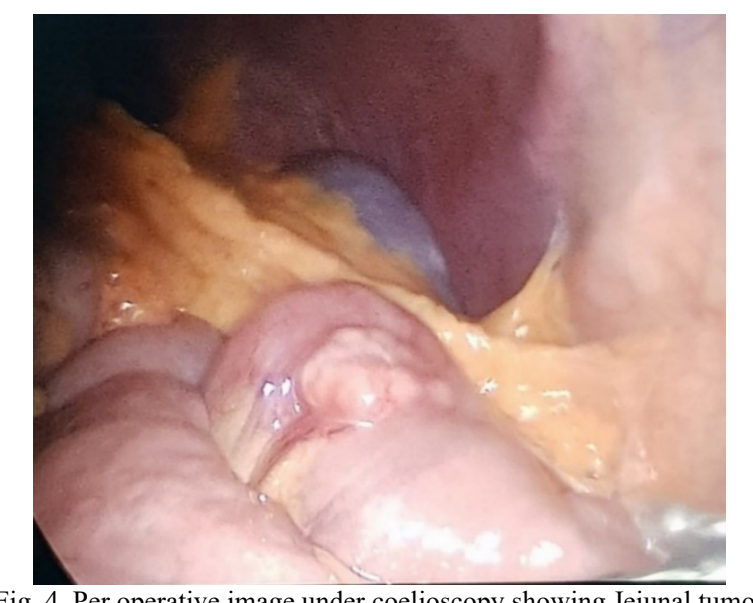

Fig. 4. Per operative image under coelioscopy showing Jejunal tumor (Red arrow).

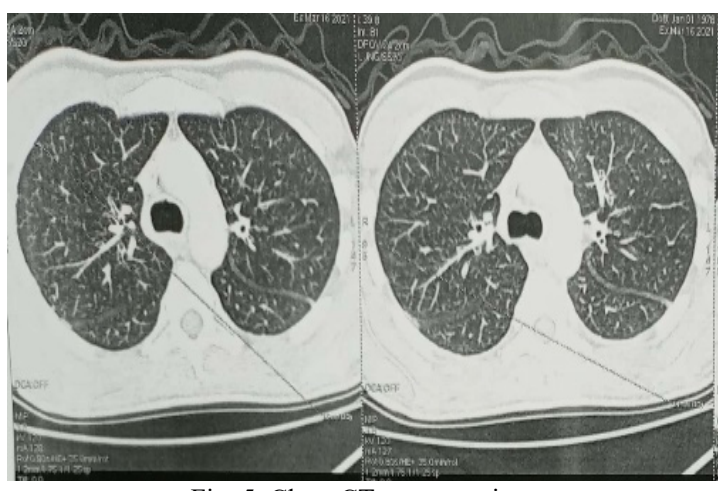

Fig. 5. Chest CT cross-section.

\section{DISCUSSION}

Malignant tumors of the small intestine are rare tumors and account for 1 to $5 \%$ of all tumors of the digestive tract. Several studies report that adenocarcinomas are the most common histologic types of cancers of the small intestine [6]. These tumors pose a diagnostic problem. Studies have shown that the median age was 46-48 years (15-80 years) [6], [7], in our case the patient aged 40 .

The presence of a predisposing pathology or a genetic syndrome appears more frequent in adenocarcinoma of small bowel than in colorectal adenocarcinomas, reaching $20 \%$ in the NADEG cohort [8].

In patients with familial adenomatous polyposis (FAP), adenocarcinomas of the duodenum and adenocarcinomas of the ampulla of Vater are the second tumor localization [8].

Individuals affected by FAP have an approximately $4.5 \%$ chance of developing SBA, which is the most common cancer-related cause of death in individuals who have had total proctocolectomy [9].

The relative risk of developing adenocarcinoma of the small intestine in case of HNPCC syndrome is high, 291 in case of hMLH1 mutation and 103 in case of hMSH2 mutation in the Dutch registry [10].

Apart from a genetic predisposition, adenomas of the small intestine, in case of large size, villous contingent or periampullary localization, also present a risk of degeneration [10].

The standardized relative risk of developing small bowel adenocarcinoma compared to the general population is 34 for Crohn's disease involving the small intestine and 46 if the course is longer than 8 years. Dysplasia surrounds the adenocarcinoma in $49 \%$ of cases [8].

Coeliac disease is characterized by a lymphocytic infiltrate that induces immunological disruption and damage to the epithelial cells that can include premalignant changes and could increase the risk of both SBA and small bowel lymphoma [8].

Celiac disease is associated with a relative risk of adenocarcinoma of the small intestine compared to the general population which is estimated at 10 in a Swedish registry study [8].

In our patient an etiological assessment was carried out, including upper digestive endoscopy and colonoscopy, with biopsies searching for Crohn's disease, celiac disease and hereditary polyposis showed no abnormalities. Fecal calprotectin and anti-transglutaminase antibodies were also negative.

The clinical manifestations are variable and not very specific which leads to a delay in diagnosis, the tumor can present by melena, abdominal mass, or abdominal occlusion. The average duration of symptoms is 4 to 5 months [2].

A study conducted at the Department of Gastrointestinal Surgery, Kunshan First Peoples Hospital Affiliated with Jiangsu University, Kunshan in 2015 presents a case of a patient with duodenal obstruction suspected by the passage of the intestinal loop through the duodeno-mesenteric space, however, macroscopic examination revealed a type 2 tumor extension with serous infiltration at the duodeno-jejunal junction, which resulted in complete bowel obstruction Prompt histological diagnosis during surgery revealed moderately differentiated adenocarcinoma [3].

CT enterography, video-capsule, or enteroscopy allow better exploration of tumors of small intestine; with the possibility of taking biopsies by enteroscopy [2].

The curative treatment is surgical resection. Small bowel tumors have bad prognosis due to its vague symptoms leading to delayed diagnosis. [4], [8].

\section{CONCLUSION}

Small bowel adenocarcinoma is a rare pathology, Diagnosis of SBA remains a challenge. A physician's suspicion and awareness are crucial in patients with abdominal pain of unknown cause to avoid delayed diagnosis and subsequent poor prognosis.

\section{REFERENCES}

[1] Ofori E, Ramai D, Papafragkakis C, Changela K, Krishnaiah M. Primary Jejunal Adenocarcinoma Presenting as Bilateral Ovarian Metastasis. Gastroenterol Res. 2017;10(6):366-8.

[2] Vilde F, Wdowik A. Adénocarcinome du jé junum: apport de l'enteroscopie poussée. A propos d'un cas. Acta Endosc. 1996;26:5.

[3] Chang H-K, Yu E, Kim J, Bae YK, Jang K-T, Jung ES, et al. Adenocarcinoma of the small intestine: a multi-institutional study of 197 surgically resected cases. Hum Pathol. août 2010;41(8):1087-96.

[4] Zaanan A, Afchain P, Carrere N, Aparicio T. Adénocarcinome de l'intestin grêle. Gastroentérologie Clin Biol. août 2010;34(6-7):371-9.

[5] Delaunoit T, Neczyporenko F, Limburg PJ, Erlichman C. Small Bowel Adenocarcinoma: A Rare but Aggressive Disease. Clin Colorectal Cancer. nov 2004;4(4):241-8.

[6] Halima A, Maha M, Issam L, Hind M, Hassan E. Les tumeurs malignes primitives de l'intestin grêle: aspects cliniques et thérapeutiques de 27 patients:7.

[7] Mellouki I, Jellali K, Ibrahimi A. Les tumeurs du gréle: à propos de 27 cas. Pan Afr Med J [Internet]. 2018 [cité 25 avr 2021];30. Disponible 
sur: http://www.panafrican-med-

journal.com/content/article/30/13/full/.

[8] Aparicio T. Small bowel adenocarcinoma: Epidemiology, risk factors, diagnosis and treatment. Dig Liver Dis. 2014;8.

[9] Katrina S. Pedersen, MD, MSa; Kanwal Raghav, MBBS, MDb; and Michael J. Overman, MDb. Small Bowel Adenocarcinoma: Etiology,
Presentation, and Molecular Alterations in: Journal of the National Comprehensive Cancer Network Volume 17 Issue 9 (2019).

[10] Chapitre 13: Adénocarcinome de l'intestin grêle. Thésaurus national de cancérologie digestive. 2017. 